\title{
M4 Anhang M: Diagramme und Tabellen
}

C. Hainbach, Essen; S. Schädlich, Raesfeld, Bottrop

Anh. M1 Tabelle 1. Klimadaten nach M1 [1]

\begin{tabular}{|c|c|c|c|c|c|c|c|c|}
\hline \multirow[t]{2}{*}{ Gebiet und Ort } & \multirow[t]{2}{*}{$\begin{array}{l}\text { Seehöhe } \\
\text { in } \mathrm{m}\end{array}$} & \multicolumn{3}{|c|}{ Mittlere Temperatur ${ }^{\circ} \mathrm{C}$} & \multicolumn{2}{|c|}{ Extreme Temperatur ${ }^{\circ} \mathrm{C}$} & \multicolumn{2}{|c|}{$\begin{array}{l}\text { Mittlere relative Luft- } \\
\text { feuchtigkeit in } \%\end{array}$} \\
\hline & & im Jahr & $\begin{array}{l}\text { im wärmsten } \\
\text { Monat }\end{array}$ & $\begin{array}{l}\text { im kältesten } \\
\text { Monat }\end{array}$ & maximal & minimal & maximal & minimal \\
\hline \multicolumn{9}{|l|}{ Europa } \\
\hline Kopenhagen & 10 & 7,7 & $16,6 \mathrm{Jl}$ & $-0,1 \mathrm{~F}$ & 29 & -13 & $93 \mathrm{D}$ & $72 \mathrm{Ju}$ \\
\hline Helsinki & 10 & 4,4 & $16,6 \mathrm{Jl}$ & $-6,9 \mathrm{~F}$ & 26 & -24 & $89 \mathrm{~N}$ & $68 \mathrm{Jn}$ \\
\hline Dublin & 10 & 9,9 & $15,7 \mathrm{Jl}$ & $5,3 \mathrm{Jr}$ & 25 & -5 & $86 \mathrm{Jr}$ & $73 \mathrm{Ma}$ \\
\hline London & 40 & 9,8 & $17,3 \mathrm{Jl}$ & $3,4 \mathrm{Jr}$ & 31 & -8 & $89 \mathrm{Jr}$ & $69 \mathrm{Jl}$ \\
\hline Paris & 50 & 10,3 & $18,6 \mathrm{Jl}$ & $2,5 \mathrm{Jr}$ & 34 & -11 & $89 \mathrm{D}$ & 69 Ap \\
\hline Hamburg & 30 & 8,3 & $16,9 \mathrm{~J} 1$ & $-0,3 \mathrm{Jr}$ & 30 & -12 & $90 \mathrm{D}$ & $69 \mathrm{Ma}$ \\
\hline Frankfurt a.M. & 100 & 9,5 & $18,6 \mathrm{Jl}$ & $0,1 \mathrm{Jr}$ & 33 & -13 & $86 \mathrm{D}$ & $66 \mathrm{Ma}$ \\
\hline Wien & 200 & 9,2 & $19,6 \mathrm{~J} 1$ & $-1,7 \mathrm{Jr}$ & 30 & -15 & $85 \mathrm{D}$ & $67 \mathrm{Ap}$ \\
\hline Berlin & 40 & 8,6 & $18,0 \mathrm{Jl}$ & $-0,7 \mathrm{Jr}$ & 32 & -15 & $87 \mathrm{D}$ & $65 \mathrm{Jn}$ \\
\hline Warschau & 120 & 7,3 & $18,8 \mathrm{Jl}$ & $3,4 \mathrm{Jr}$ & 32 & -21 & $89 \mathrm{D}$ & $68 \mathrm{Ma}$ \\
\hline Leningrad & 10 & 3,7 & $17,7 \mathrm{Jl}$ & $-9,3 \mathrm{Jr}$ & 29 & -29 & $89 \mathrm{D}$ & $65 \mathrm{Jn}$ \\
\hline Moskau & 140 & 3,9 & $18,9 \mathrm{Jl}$ & $-11,0 \mathrm{Jr}$ & 31 & -31 & $87 \mathrm{D}$ & $67 \mathrm{Ma}$ \\
\hline Astrachan & -10 & 9,4 & $25,5 \mathrm{Jl}$ & $-7,2 \mathrm{Jr}$ & 36 & -26 & $75 \mathrm{D}$ & $32 \mathrm{Au}$ \\
\hline Bukarest & 80 & 10,4 & $22,8 \mathrm{Jl}$ & $-3,6 \mathrm{Jr}$ & 35 & -20 & $87 \mathrm{D}$ & $60 \mathrm{Au}$ \\
\hline Istanbul & 70 & 14,5 & $23,6 \mathrm{Au}$ & $5,2 \mathrm{Jr}$ & 34 & -4 & $74 \mathrm{D}$ & $53 \mathrm{Jl}$ \\
\hline Athen & 110 & 17,7 & $27,0 \mathrm{Jl}$ & $8,3 \mathrm{Jr}$ & 38 & -2 & $75 \mathrm{D}$ & $46 \mathrm{Au}$ \\
\hline Bozen & 290 & 11,7 & $22,5 \mathrm{Jl}$ & $0,0 \mathrm{Jr}$ & 34 & -8 & $83 \mathrm{~N}$ & $61 \mathrm{Ap}$ \\
\hline Rom & 50 & 15,4 & $24,8 \mathrm{Jl}$ & $6,7 \mathrm{Jr}$ & 35 & -3 & $74 \mathrm{D}$ & $53 \mathrm{Jl}$ \\
\hline Madrid & 650 & 13,3 & $24,3 \mathrm{Jl}$ & $4,3 \mathrm{Jr}$ & 40 & -8 & $84 \mathrm{D}$ & $46 \mathrm{Jl}$ \\
\hline \multicolumn{9}{|l|}{ Afrika } \\
\hline Tripolis & 20 & 19,7 & $26,4 \mathrm{Au}$ & $11,7 \mathrm{Jr}$ & 40 & 4 & $67 \mathrm{Jl}$ & $63 \mathrm{~S}$ \\
\hline Marrakesch & 470 & 19,6 & $29,6 \mathrm{Au}$ & $10,9 \mathrm{Jr}$ & 41 & 3 & $66 \mathrm{Jr}$ & $47 \mathrm{Jl}$ \\
\hline Dakar & 20 & 24,2 & $28,0 \mathrm{~S}$ & $20,3 \mathrm{~F}$ & 34 & 15 & $87 \mathrm{Au}$ & $81 \mathrm{~F}$ \\
\hline Kapstadt & 10 & 16,4 & $20,7 \mathrm{Jr}$ & $12,2 \mathrm{Jl}$ & 34 & 4 & $81 \mathrm{Jn}$ & $66 \mathrm{Jr}$ \\
\hline Johannesburg & 1920 & 14,6 & $18,5 \mathrm{Jr}$ & $9,0 \mathrm{Jn}$ & 30 & -3 & $74 \mathrm{~F}$ & $39 \mathrm{Au}$ \\
\hline Dar es Salam & 10 & 25,5 & $27,7 \mathrm{Jr}$ & $23,1 \mathrm{Au}$ & 33 & 17 & $85 \mathrm{Ap}$ & $79 \mathrm{Jr}$ \\
\hline \multicolumn{9}{|l|}{ Asien } \\
\hline Jerusalem & 750 & 15,9 & $23,0 \mathrm{Au}$ & $7,0 \mathrm{Jr}$ & 36 & -2 & $74 \mathrm{Jr}$ & $41 \mathrm{Ma}$ \\
\hline Taschkent & 480 & 13,5 & $27,5 \mathrm{Jl}$ & $-1,0 \mathrm{Jr}$ & 40 & -20 & $77 \mathrm{Jr}$ & $48 \mathrm{Jl}$ \\
\hline Peking & 40 & 11,7 & $26,0 \mathrm{Jl}$ & $-4,7 \mathrm{Jr}$ & 37 & -15 & $76 \mathrm{Au}$ & 49 Ap \\
\hline Hongkong & 30 & 22,0 & $27,6 \mathrm{Jl}$ & $14,3 \mathrm{~F}$ & 36 & 6 & $84 \mathrm{Ap}$ & $65 \mathrm{~N}$ \\
\hline Tokio & 20 & 13,8 & $25,4 \mathrm{Au}$ & $2,9 \mathrm{Jr}$ & 37 & -9 & $84 \mathrm{Jl}$ & $63 \mathrm{~F}$ \\
\hline Delhi & 220 & 25,1 & $33,4 \mathrm{Jn}$ & $14,4 \mathrm{Jr}$ & - & - & $68 \mathrm{Au}$ & $33 \mathrm{Ap}$ \\
\hline Bombay & 10 & 26,3 & $29,2 \mathrm{Ma}$ & $23,6 \mathrm{Jr}$ & 35 & 16 & $87 \mathrm{Au}$ & $69 \mathrm{~F}$ \\
\hline Singapur & 0 & 26,3 & $27,0 \mathrm{Ma}$ & $25,5 \mathrm{Jr}$ & 38 & 18 & $84 \mathrm{D}$ & $78 \mathrm{Jl}$ \\
\hline Djakarta-Java & 10 & 26,0 & $26,5 \mathrm{O}$ & $25,8 \mathrm{Jl}$ & 33 & 20 & $87 \mathrm{Jl}$ & $78 \mathrm{~S}$ \\
\hline \multicolumn{9}{|c|}{ Australien, Ozeanien } \\
\hline Adelaide & 40 & 17,2 & $23,4 \mathrm{Jr}$ & $10,9 \mathrm{Jl}$ & 43 & 2 & $77 \mathrm{~J} 1$ & $46 \mathrm{Jr}$ \\
\hline Auckland & 80 & 15,2 & $19,6 \mathrm{~F}$ & $11,1 \mathrm{Au}$ & 29 & 2 & $82 \mathrm{Jl}$ & $73 \mathrm{Jr}$ \\
\hline \multicolumn{9}{|l|}{ Amerika } \\
\hline Winnipeg & 230 & 0,6 & $18,7 \mathrm{Jl}$ & $-21,7 \mathrm{Jr}$ & 33 & -40 & $90 \mathrm{Jr}$ & $69 \mathrm{Ma}$ \\
\hline Washington & 40 & 12,6 & $24,9 \mathrm{Jl}$ & $0,5 \mathrm{Jr}$ & 36 & -16 & $79 \mathrm{~S}$ & 64 Ap \\
\hline Chikago & 250 & 9,2 & $22,4 \mathrm{Jl}$ & $-4,6 \mathrm{Jr}$ & 35 & -26 & $81 \mathrm{Jr}$ & $67 \mathrm{Jl}$ \\
\hline Los Angeles & 110 & 15,7 & $20,3 \mathrm{Au}$ & $11,7 \mathrm{Jr}$ & 38 & 1 & $76 \mathrm{Au}$ & $67 \mathrm{~N}$ \\
\hline Mexico & 2280 & 15,5 & $18,3 \mathrm{Ma}$ & $11,9 \mathrm{D}$ & 30 & 1 & $71 \mathrm{~S}$ & $47 \mathrm{Ap}$ \\
\hline Habana-Cuba & 20 & 24,8 & $27,7 \mathrm{Jl}$ & $21,3 \mathrm{Jr}$ & 36 & 12 & $80 \mathrm{~S}$ & $70 \mathrm{Ap}$ \\
\hline Valparaiso & 40 & 14,3 & $17,5 \mathrm{~F}$ & $11,5 \mathrm{Jl}$ & 28 & 6 & $78 \mathrm{Ma}$ & $66 \mathrm{D}$ \\
\hline Buenos Aires & 20 & 16,6 & $23,1 \mathrm{Jr}$ & $10,1 \mathrm{Jl}$ & 34 & 0 & $86 \mathrm{Jn}$ & $70 \mathrm{D}$ \\
\hline Rio de Janeiro & 60 & 22,5 & $25,6 \mathrm{~F}$ & $19,7 \mathrm{Jl}$ & 36 & 13 & $80 \mathrm{~F}$ & $77 \mathrm{Jl}$ \\
\hline
\end{tabular}

Jr Januar. - F Februar. - Ma März. - Ap April. - Jn Juni. - Jl Juli. - Au August. - S September. - O Oktober. - N November. - D Dezember. 